\title{
Multidimensional scaling of subjective colors by color-blind observers
}

\author{
CHARLES W. WHITE \\ New School for Social Research, New York, New York 10003 \\ and \\ GREGORY R. LOCKHEAD and NANCY J. EVANS \\ Duke University, Durham, North Carolina 27706
}

\begin{abstract}
Temporal coding theories of color vision suggest explanations of flicker-induced subjective colors such as those that appear on Benham's disk. If color blindness were due simply to photopigment anomalies, then subjective colors might be elicited by central patterns of neural activity in color-blind observers that mimic those which the cones normally produce in colornormal observers. We had color-normal and color-blind observers scale subjective colors like those on Benham's disk for similarity. The inferred color spaces for six normal observers resembled the familiar hue circle, but the spaces for five red-green-deficient observers were compressed along the red-green axis. This is consistent with the position that flicker colors are due to retinal processes, and suggests that color blindness may involve variations of the central nervous system in addition to photopigment anomalies.
\end{abstract}

Current theories assume that the initial stage of color vision involves three types of cones in the retina. Cones provide the input to subsequent neural stages that determine colors by opponent processes. The colorencoding mechanisms have been mapped with such precision in the last decade that ample evidence now exists to support a fusion of the 19th century color-vision theories of Helmholtz and Hering associated with these processes. However, the physiological system so far described does not explain how color information is processed in the human visual system. There remain many phenomena which current theories of color vision fail to explain. An important example is that various black and white patterns, such as Benham's disk (in Figure 1), appear colored when they are flashed or rotated at appropriate rates. The colors that appear are called subjective colors.

\section{Subjective Colors}

There is a widespread general awareness of subjective colors [cf. Cohen and Gordon's (1949) review of the early work], but there have been few attempts to specify how those colors are related to normal colors. Piéron (1952) suggested an explanation in terms of differential rise and fall times for the three primary color sensations. Without modification, this explanation now appears inadequate. It demands much greater differences in laten-

Supported in part by a grant to the first author from the Duke University Research Council. Request reprints from Charles W. White, The Graduate Faculty, Department of Psychology, Program in Visual Perception, New School for Social Research, 65 Fifth Avenue, New York, New York 10003. cies between the primary color sensations than have been observed (Green, 1969; Guth, 1964; Marrocco, 1976).

Recently, Campenhausen (1969) and Festinger, Allyn, and White (1971), among others, have revived an old idea that color information may be encoded within the visual system as temporal patterns of neural activity. Fry (1933) and Troland (1921) had suggested that all color information might be conveyed by differential neural firing patterns. Temporal coding theories of color explain subjective colors as follows: The local luminance fluctuations produced by a rotating Benham's disk produce temporal patterns of neural activity in the visual

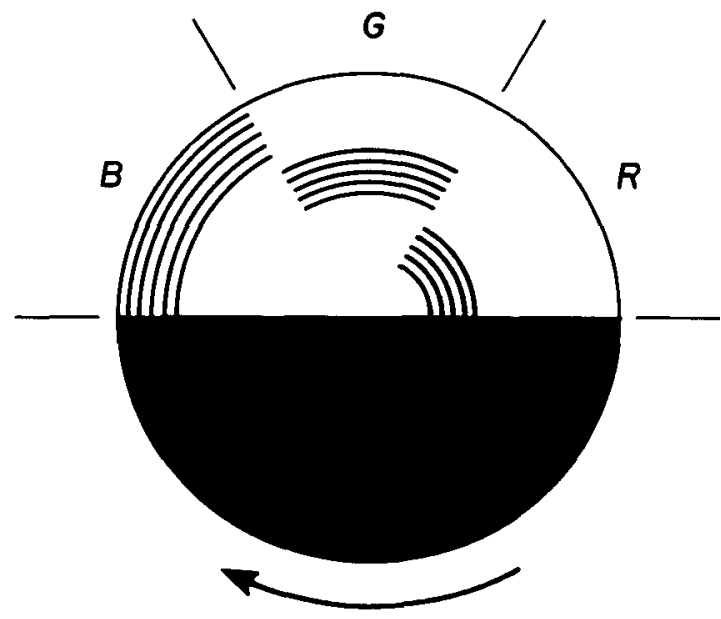

Figure 1. Benham's disk. The arcs in sectors, $R, G$, and $B$ normally appear redish, greenish, and bluish when the disk is rotated clockwise at approximately $\overline{7}$ revolutions/sec. $B$ and $R$ exchange colors when the disk rotates counterclockwise. 
system. Certain patterns correspond in some way to the temporal neural patterns that are ordinarily produced by the peripheral color-encoding mechanisms. To the extent that responses to Benham's disk mimic those temporal patterns, the flickering black-and-white disk appears colored.

\section{Color-Blindness}

Temporal coding theories of color vision suggest an interesting possible relationship between color blindness and subjective colors. If color blindness is due to a photopigment anomaly, as is often assumed, then subjective colors might be able to elicit patterns of neural activity that mimic those which the cones normally produce. The temporal patterns provided by the flickering pattern might substitute for the normal temporal pattern. Thus, subjective colors might bypass the colordeficient stage of the color-blind visual system. It might even be possible to create, for a color-blind observer, a color which he had not previously experienced.

It is difficult to assess the subjective colors seen by observers who have color-deficient vision. Color names are predictably unreliable, given a lifetime of learning to make arbitrary distinctions among indistinguishable hues. Colorimetric matches by color-blind observers are also too variable. Moreover, if a subjective color did appear novel, the observer would be unable to match it with any mixture of wavelengths.

Comments such as "That line is definitely colored, but I don' $t$ know what to call it" and "Those lines are greener than grass" were recorded by one of us (C.W.W.) from color-blind observers viewing a Benham disk. Such comments suggest that the observers were viewing something unusual or unique. We must be cautious, however. The novel color descriptions could simply be responses to the novelty of the flickering aspects of the disk, or to the motions of the patterns, or to something else.

Perhaps because of measurement difficulties, we know of only three previous reports mentioning subjective colors as viewed by color-deficient observers: (1) Stewart (1924) reported an observer who had been told previously that his color perception was abnormal and who did not perceive the colors seen by normal viewers in a situation similar to that of the Benham disk; (2) a brief comment suggested that protanopic observers matched red on a Benham disk with Munsell colors of higher values (i.e., lighter) than did most color normals (Anonymous, 1968); (3) Land (1975) noted general agreement in the reported perceptions of deuteranomalous observers and color-normal observers when viewing a Benham disk through colored filters.

\section{Color Scaling}

Shepard and Cooper (Note 1) reported the multideminsional similarity scaling of surface colors by color-deficient observers. When red-green-deficient observers made similarity judgments of pairs of colored chips, the derived hue circle was compressed along the red-green axis, in accordance with the chromaticity confusion lines for protanopia and deuteranopia. But when the same observers scaled a set of color names, the hue circle in the multidimensional space resembled results of color-normal observers. Carroll and Chang (Note 2) reported similar observations. Shepard and Cooper concluded that the color-deficient observers had a normal conception of the relationships among colors.

Since similarity scaling produces results consistent with those from discrimination tasks, color-blind observers should be able to scale subjective colors by judging the similarity of various pairs of stimuli, and the color spaces derived from the scaling procedure should indicate the relationships among the subjective colors they perceive. We conducted the following experiment to compare the scaling of some subjective colors by color-deficient and by color-normal observers.

\section{METHOD}

\section{Observers}

The six color-normal observers were graduate students or faculty members, two females and four males. The six colorblind observers were undergraduate and graduate students, five males and one female.

The color anomalous observers were tested with the H-R-R pseudoisochromatic plates (Hardy, Rand, \& Rittler, 1957) using the recommended standard light source and viewing distance. According to the test criteria, they were classified as three medium protans, one medium deutan, and two strong deutans.

\section{Stimuli}

The apparatus consisted of paper patterns wrapped around a metal cylinder, $11.4 \mathrm{~cm}$ high and $6.4 \mathrm{~cm}$ in diameter, mounted on a vertical motor shaft. The cylinder was raised $9 \mathrm{~cm}$ above a table top by the motor housing and was illuminated by two $15-W$ cool-white fluorescent lamps in a reflector housing $38 \mathrm{~cm}$ directly above the cylinder.

The observers sat in front of the table and viewed the cylinder at eye level from a distance of $46 \mathrm{~cm}$. The cylinder was rotated at a constant rate of 7 revolutions/sec.

The subjective color patterns were constructed on heavy nonglare white paper and were wrapped around the rotating cylinder. One-half of the cylinder was covered by matte-black Mylar film. The thin black lines on the other half of the pattern were made from $0.8-\mathrm{mm}$ matte-black chart tape. The length and position of the line segments were varied to make the eight different stimuli that are shown in Figure 2. Three of the stimuli, the lines labeled red, light green, and blue, are the rectilinear equivalents of the $60^{\circ}$ arcs shown on the disk in Figure 1. The other five lines were intended to increase the variety of subjective colors available in the display. Some of the lengths and positions were based loosely on suggestions by Festinger et al. (1971) and Polizzotto and Peura (1975), but all were chosen to produce the widest range of colors possible for one of the authors (N.J.E.).

The patterns used for similarity judgments combined the stimuli shown in Figure 2. The eight stimuli were paired to provide all 28 possible combinations of each pattern paired with every other pattern. The pairs were randomly divided into three groups of either 9 or 10 pairs each, and a pattern to be mounted on the cylinder was constructed for each group. The lines within each pair were separated vertically by $3 \mathrm{~mm}$. Pairs were separated on the cylinder by 9 or $10 \mathrm{~mm}$, depending on whether the total pattern contained 9 or 10 pairs. The arrangement of the members of each line pair was determined randomly. 


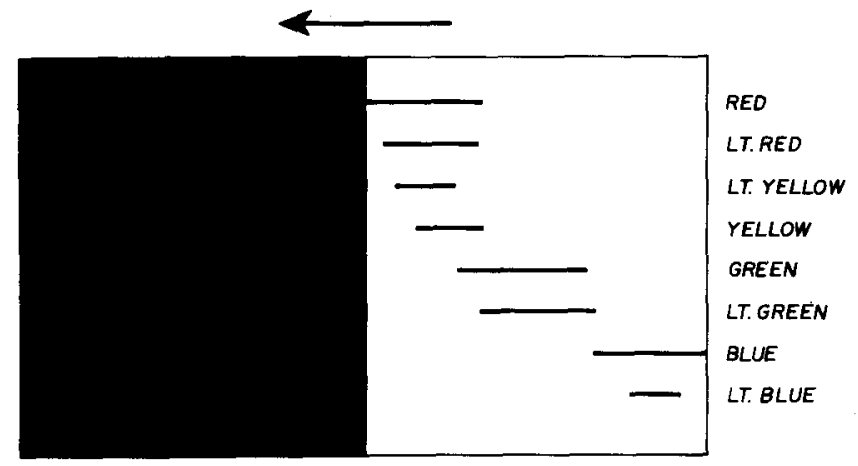

Figure 2. Subjective color patterns. The eight stimuli on this pattern were combined in pairs for similarity scaling. The mnemonic color names are based on color naming and on Munsell matches by color-normal observers.

\section{Procedure}

Each observer sat in front of the apparatus and the first pattern was placed on the cylinder, which was then brought up to speed. The observer was told to describe the appearance of the lines while the cylinder was reaching its steady-state rate of rotation. If no colors were reported immediately, the observer was told that colors would probably appear as the speed of the drum increased. All observers reported seeing colors after a minute or two of observation.

The observers were instructed to assign numbers to describe the similarity of colors within each of the line pairs, with " 1 " representing "very similar" and " 6 " representing "very dissimilar." They assigned a number to each pair on the cylinder, going from top to bottom. They were told to look away for a few minutes and then repeat the scaling procedure, this time from bottom to top. They looked away as the cylinder was changed, and the procedure was repeated for the remaining two patterns.

\section{RESULTS}

\section{Color-Normal Observers}

To aid in interpreting the similarity results, each of the authors independently selected that Munsell color which best matched each of the eight subjective colors used in the study. The subjective colors were generated by reproducing the line arrangements of Figure 2 on a cylinder. Matching was accomplished by selecting the best fitting patch from an opposite hue edition of Munsell colors which contains the full range of Munsell papers. The cylinder and the book were flooded by the same illuminant as used in the experiment.

The Munsell matches were converted to CIE tristimulus values, and the eight matches were averaged over the three observers. The resulting estimate of the appearance of these subjective colors is shown in CIE chromaticity coordinates in Figure 3. In color names, these eight colors might be labeled as: red, light red, light yellow, yellow, green, light green, blue, and light blue.

The similarity judgments given to each pair of subjective colors by the color-normal observers were averaged over the six observers. The resulting similarity matrix was scaled by the Guttman-Lingoes nonmetric method. The smallest space coordinates for the onedimensional solution account for $78 \%$ of the variance in the judgments, the two-dimensional solution accounts for $92 \%$ of the variance, and the three-dimensional solution accounts for $98 \%$ of the variance. The onedimensional solution is judged not to be an adequate fit; at least two dimensions are required to account for the similarity judgments of the eight subjective colors used in this study.

Visual comparisons of Figure 3 and the upper panel of Figure 4 show that these are very similar spaces. The three-dimensional scaling solution of the subjective color similarities, for color-normal observers, produces a similarity space which is essentially identical with that obtained when observers match those subjective colors with surface colors.

\section{Color-Deficient Observers}

One observer, a medium protan, reported scaling on the basis of "how bright things are" and his results are very different from those of all of the 11 other subjects in the study. This observer described the green-light red pair of subjective colors as "green-brown and orange," but he called the green-red pair (same green) "purple and orange." It appears he was seeing different hues, but there is no strong indication that these hue differences matched those of any of the other observers. The results of this observer were not included in the averaged analysis reported here.

Similarity judgments of each stimulus pair were averaged over the remaining five observers. As with the color-normal data, the resulting similarity matrix was scaled by the Guttman-Lingoes nonmetric method. The one-dimensional solution accounts for $93 \%$ of the variance, two dimensions account for $97 \%$ of the variance, three dimensions account for $99 \%$ of the variance. Although the one-dimensional solution is judged to be an adequate fit, the three-dimensional solution for

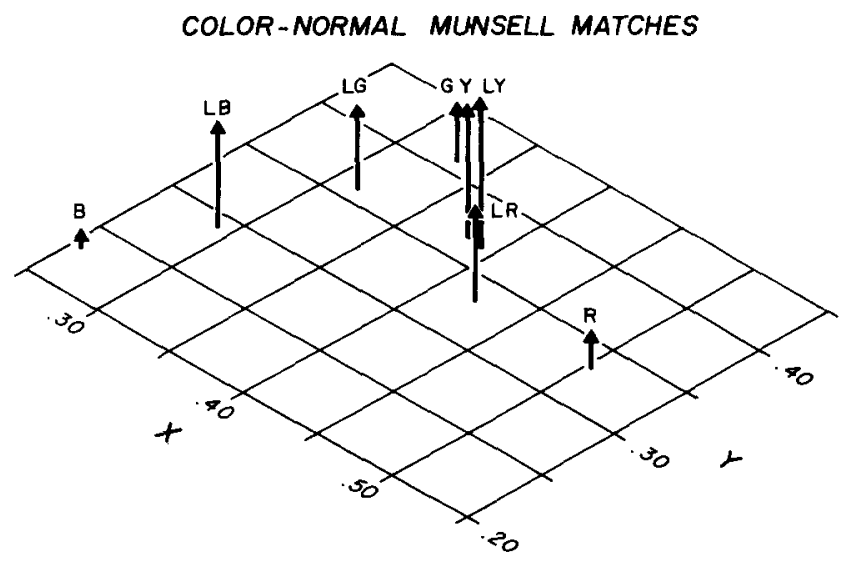

Figure 3. Average location of the Munsell matches of the eight subjective colors displayed on a section of the CIE $x, y$ diagram. The heights of the arrows are proportional to the luminance of the matching chips. Letters identify the stimuli in Figure 2. 
the color-deficient observers is shown in the lower panel of Figure 4 to aid in comparison of these data with those from the color-normal observers. The two spaces in Figure 4 are principally different in that the reds are judged essentially identical to the greens by the colordeficient observers, and very different from the greens by the color-normals.

The similarity data for each of the five color-deficient observers reported were scaled separately. Onedimensional solutions account for more than $90 \%$ of the variance for each of the observers, except for Observer 1 . The one-dimensional solution accounts for $86 \%$ of the variance in this observer's similarity matrix, two dimensions account for $94 \%$. The one-dimensional solutions for each of the five color-deficient observers, the solution for their average similarity matrix, and (for comparison) the poor-fitting one-dimensional average solution for the color-normals, are shown together in Figure 5. The one-dimensional solution for Observer 5 degenerated into two groups. In a metric analysis of his similarities, in which judgments are treated directly as
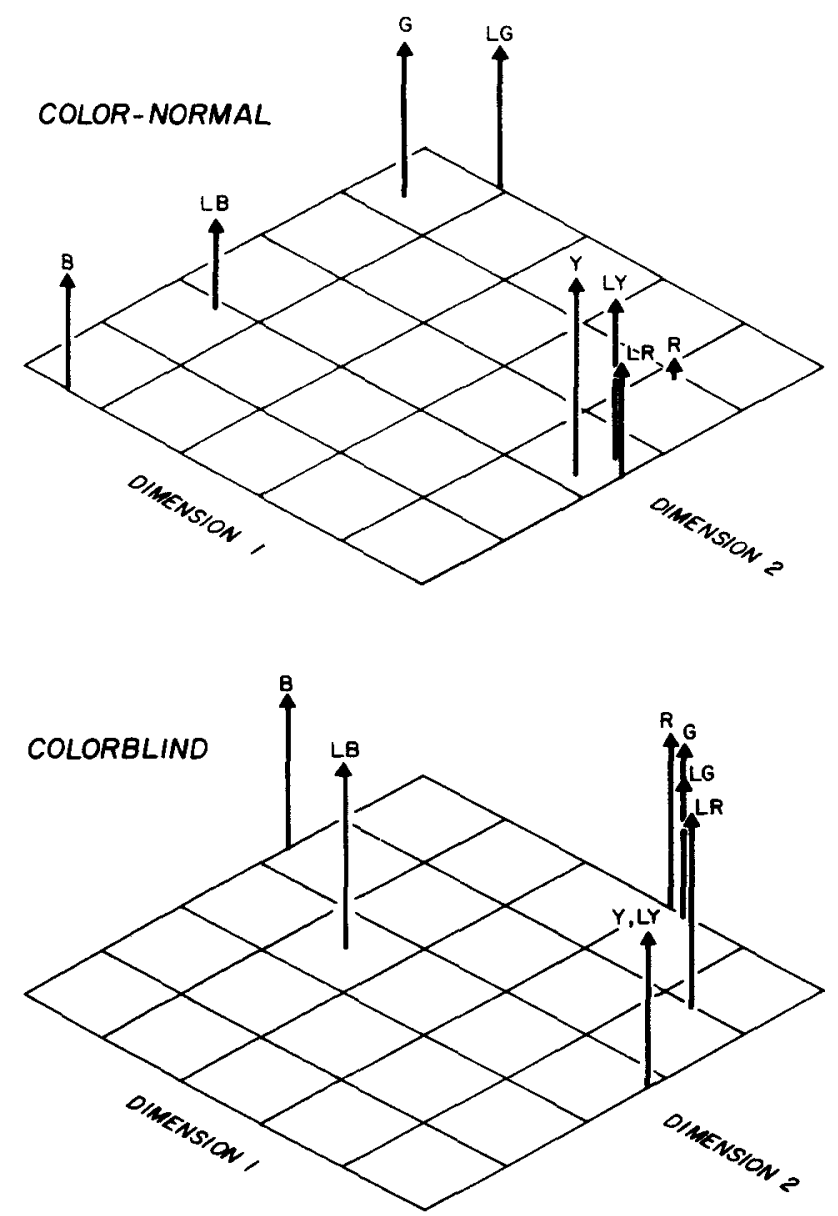

Figure 4. Three-dimensional similarity space for the colornormal observers (upper panel) and the color-blind observers (lower panel). Line lengths show distance out of the reference plane; the stimuli are located at the arrow points.
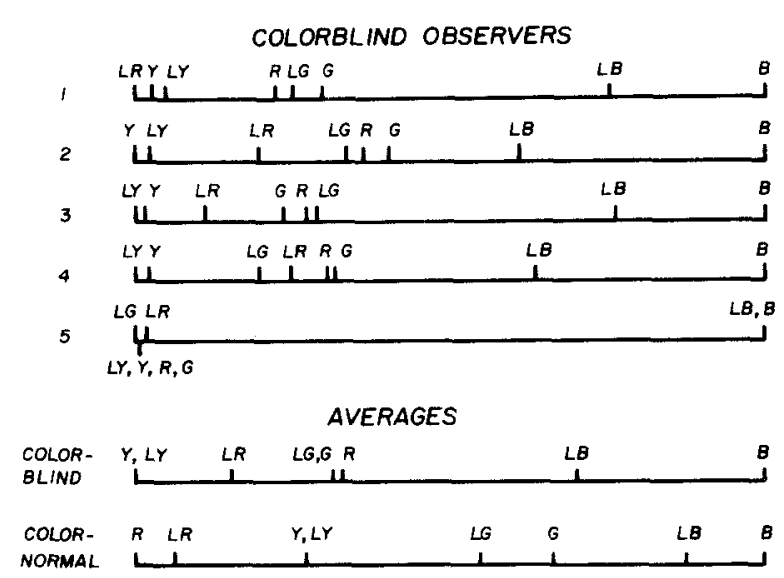

Figure 5. One-dimensional similarity scales for individual color-blind observers; Observers 1, 2, and 3 are deutans, 4 and 5 are protans. Average scales for color-deficient and color-normal observers are shown at the bottom.

distances rather than as ranks, this observer's data looks like those of the other observers in that yellows are at one end of the scale and the red and greens move together towards the blues, which are at the other end of the scale. We use the nonmetric analysis in this paper because the necessary assumptions are more conservative.

Figure 5 shows that each of the color-deficient observers placed the yellows and blues at the extremes of the scale and that red is-for every observer-more like both greens than it is like either yellow. Since there are two lightnesses of yellow and of green, and since the red is placed with the greens independent of lightness, it appears that this similarity judgment is made on the basis of hue. The color-normal observers, on the other hand, place the yellows between the reds and greens, and have red and blue as the scale extremes. There are no obvious differences in the data between protans and deutans.

\section{DISCUSSION}

Color-blind observers see subjective colors just as they see other colors; we were not successful in making new colors for them. What are subjective red and green to color-normals, and thus very different, are very similar to protans and deutans. What are subjective yellow and blue to color-normals, and thus again very different, are also very different to color-blinds. In terms of Benham's disk (Figure 1), protans and deutans discriminate arc B from arcs $R$ and $G$, but do not differentiate $R$ from $G$.

Temporal coding theories allowed the suggestion that flickering patterns might provide temporal codes which color-blinds, because of their cone pigment deficiency, do not ordinarily see. Color-blinds might thus see colors they have never experienced before if we bypass this temporal patterning mechanism and provide the code directly. This is not supported by our data; the temporal 
patterns provided by the flickering stimuli do not successfully substitute for those temporal patterns missing because of pigment anomalies.

If we have provided appropriate temporal patterns, and thus bypassed the normal code-inducing mechanisms, we are forced to the conclusion that something more than photopigments is different between color-normals and color-blinds. It is as if color-normals have a temporally coded color system which is not available to color-blinds. Continuing the speculation, this is consistent with observations from developmental research. For example, it is reported that animals deprived of vertical line stimulation do not develop vertical line detectors (Hirsch \& Spinelli, 1970). Should different cone responses in color-normals set up a temporal code which stimulated part of the visual system, the analog of a color detector may develop. Color-blinds, because of missing or replaced photopigments, have not had the appropriate temporal code stimulation during development and did not develop all color detectors.

By this suggestion, color-blind observers do not differ from normals just in terms of photopigments or cones; not having normal photopigments results in aspects of the central visual system that are also different from those of color-normals.

Alternatively, we may not have bypassed the normal inducing system. If so, the receptors must generate the necessary analog to the temporal code. No mechanism for this is known, and we cannot reject Piéron's (1952) theory of different response latencies by different photopigments. Color might be due to these response differences which produce patterns of inhibition and excitation to an opponent system, or which produce lateral inhibition in the horizontal cells consistent with Campenhausen's (1969) considerations. Extension of lateral interaction to color systems is suggested by the anatomical model of Stell, Lightfoot, Wheeler, and Leeper (1975).

But, speculation aside, we must again conclude that something other than photopigments is different between the two populations. Otherwise, our study would have allowed color-blinds to discriminate red from green. The simplest difference to imagine is that the type of neural connection from a photopigment is determined by that photopigment; all red-sensitive cones have the same neural result. In the color-blind, whose "red" pigment is replaced by "green," the same neural response results from both receptors, while the color-normal has access to different responses from each of them.

Whatever the differences between color-normals and color-blinds, whether an accompaniment of the genetically determined cone differences, or a developmentally induced difference due to lack of appropriate temporal codes early in life, we conclude that something in addition to photopigment differences discriminates color-blinds from color-normals.

This experiment also extends the observation that similarity scaling is a useful tool for determining relations between perceptions which have historically proved difficult to obtain. What discriminations colorblinds can make, of subjective and of other colors, has been difficult to determine when the dependent variable is a matching color. This scaling technique, which does not require that impossible task, provides reliable results and is a promising format for the study of perceptual structures.

\section{REFERENCE NOTES}

1. Shepard, R. N., \& Cooper, L. A. Representation of colors in normal. blind, and color-blind subjects. Paper presented at the meeting of the American Psychological Association, Chicago, September 1975.

2. Carroll, J. D., \& Chang, J. INDSCAL exploration of color space for normal and colordeficient subjects. Paper presented at the meeting of the American Psychological Association, Chicago, September 1975 .

\section{REFERENCES}

ANonymous. Report of the joint meeting of technical groups on vision and color, October 1967. Journal of the Optical Society of America, 1968, 58, 441.

Campenhausen, C. $v$. The colors of Benham's top under metameric illuminations. Vision Research, 1969, 9, 677-682.

Cohen, J., \& Gordon, D. A. The Prevost-Fechner-Benham subjective colors. Psychological Bulletin, 1949, 46, 97-136.

Festinger, L., Allyn, M. R., \& White, C. W. The perception of color with achromatic stimulation. Vision Research, 1971, 11, 591-612.

FRY, G. A. Modulation of the optic nerve current as a basis for color vision. American Journal of Psychology, 1933, 45, 488-492.

GrEeN, D. G. Sinusoidal flicker characteristics of the color sensitive mechanisms of the eye. Vision Research, 1969, 9, 591-604.

GuTH, S. L. The effect of wavelength on perceptual latency. Vision Research, 1964, 4, 567-578.

Hardy, L. H., Rand, G., \& Rittler, M. C. $A O H \cdot R \cdot R$ pseudoisochromatic plates. New York: American Optical Corporation, 1957.

Hirsch, H., \& SpINelli, N. Visual experience modifies distribu. tion of horizontally and vertically oriented receptive fields in cats. Science, 1970, 168, 869-871.

LAND, R. I. Monochromatic observations of pattern-induced flicker colors. Joumal of the Optical Society of America, 1975, 65, 1200. (Abstract)

MARRocco, R. T. Sustained and transient cells in monkey lateral geniculate nucleus: Conduction velocities and response properties. Journal of Neurophysiology, 1976, 39, 340-353.

Pí́Ron, H. The sensations: Their functions, processes, and mechanisms. (Trans. M. H. Pirenne \& B. C. Abbott). New Haven: Yale University Press, 1952.

Polizzotro, L., \& PeURA, R. A. A mathematical approach to explain subjective color perception. Vision Research, 1975, 15, 613-616.

Stell, W. K., Lightfoot, D. O., Wheeler, T. G., \& Leeper, H. F. Goldfish retina: Functional polarization of cone horizontal cell dendrites and synapses. Science, 1975, 190, 989-990.

Stew ART, G. N. Color phenomena caused by intermittent stimulation with white light. American Journal of Physiology, 1924, 69 , 337-353.

Troland, L. T. The enigma of color vision. American Journal of Physiological Optics, 1921, 2, 23-48.

(Received for publication September 1, 1976; accepted February 8, 1977.) 